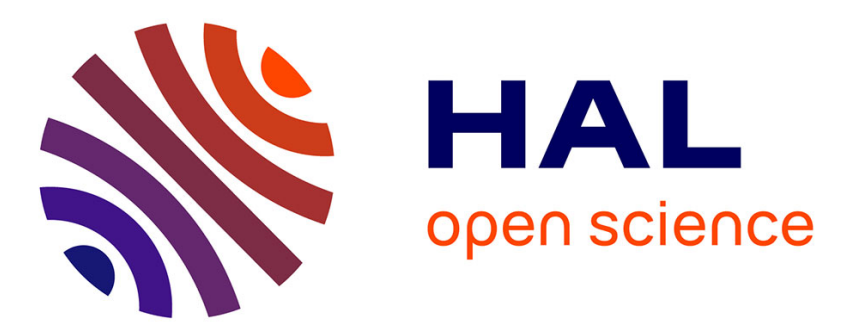

\title{
Electromagnetic Wave Propagation in the Relativistic Regime
}

\author{
J. Adam, S. Guérin, A. Héron, G. Laval, P. Mora
}

\section{To cite this version:}

J. Adam, S. Guérin, A. Héron, G. Laval, P. Mora. Electromagnetic Wave Propagation in the Relativistic Regime. Journal de Physique IV Proceedings, 1995, 05 (C6), pp.C6-1-C6-6. 10.1051/jp4:1995601 . jpa-00253964

\section{HAL Id: jpa-00253964 https://hal.science/jpa-00253964}

Submitted on 1 Jan 1995

HAL is a multi-disciplinary open access archive for the deposit and dissemination of scientific research documents, whether they are published or not. The documents may come from teaching and research institutions in France or abroad, or from public or private research centers.
L'archive ouverte pluridisciplinaire HAL, est destinée au dépôt et à la diffusion de documents scientifiques de niveau recherche, publiés ou non, émanant des établissements d'enseignement et de recherche français ou étrangers, des laboratoires publics ou privés. 


\title{
Electromagnetic Wave Propagation in the Relativistic Regime
}

\author{
J.C. Adam, S. Guérin, A. Héron, G. Laval and P. Mora \\ Centre de Physique Théorique (UPR 14 du CNRS), Ecole Polytechnique, \\ 91128 Palaiseau cedex, France
}

\begin{abstract}
An electromagnetic wave is expected to propagate through an overdense plasma, provided that its amplitude is large enough to bring the electron motion into the relativistic regime. We study this induced transparency by using $1 \mathrm{D}$ and $2 \mathrm{D}$ particle simulations. We find that large chaotic longitudinal electric fields are generated and strong plasma heating takes place during the propagation. This behavior is attributed to parametric instabilities. In order to support this interpretation, the modulational and Raman instabilities of a relativistic electromagnetic wave are studied analytically as well as with the help of particle simulations. In addition it is found that a finite width beam is strongly self focused during its propagation in a plasma layer.
\end{abstract}

\section{INTRODUCTION}

Recent progress in beam processing have increased by orders of magnitude the instantaneous power available in ultra-short laser pulses. The intensity reaches high enough levels to bring into the relativistic regime an electron interacting with the radiation. For example, the electron quiver energy reaches one $\mathrm{MeV}$ in a circularly polarized monochromatic electromagnetic wave whenever its intensity is $410^{-2} \mathrm{n}_{\mathrm{c}} \mathrm{W} / \mathrm{cm}^{2}$ where $n_{c}$ is the critical density in number of particles per $\mathrm{cm}^{-3}$. Intensities in this range have been obtained in several experimental devices [1] by using the chirped pulse amplification process.

For such extremely high intensity levels, the electromagnetic wave propagation in a plasma becomes strongly nonlinear. The electron motion is the first source of nonlinearity as the electron mass is increased by the relativistic effect and the Lorentz force is not any more negligible. Moreover, this $\mathbf{V} \times \mathbf{B}$ force induces a longitudinal electron displacement across the plane wave. Consequently, the electron density is perturbed by the wave propagation and the electric field is no longer purely transverse. Large amplitude nonlinear longitudinal oscillations are coupled to the electromagnetic wave.

nonlinear solutions of Vlasov-Maxwell equations have been obtained by several authors. They describe periodic plane nonlinear waves [2] propagating with a constant velocity in an infinite cold plasma. Other solutions provide nonlinear reflection and transmission coefficients in a bounded cold plasma.[3] The most striking feature of these waves is their ability to propagate in an overdense plasma, i.e. with a frequency lower than the plasma frequency.

Among the nonlinear plane wave periodic solutions, the most remarkable one is circularly polarized. For this wave, it is possible to write a dispersion equation and to define a refraction index $\mathrm{N}$ given by

with

$$
\mathrm{N}^{2}=1-\frac{\omega_{\mathrm{p}}^{2}}{\gamma \omega^{2}}
$$




$$
\gamma^{2}=1+\left(\frac{\mathrm{eE}}{\mathrm{m \omega c}}\right)^{2}
$$

where $E$ is the electric field amplitude and $\omega$ the wave pulsation.

For this polarization, there is no Lorentz force and consequently the electron density remains uniform. There is no longitudinal electric field. The electron velocity amplitude is constant as well as its kinetic energy. The physical meaning of Eq. (1) is easily understood by noticing that the elecron mass is increased by the transverse electron motion so that the effective plasma frequency is reduced. The plasma is transparent for $\omega_{\mathrm{p}} / \gamma \gamma<\omega$ and one may say that induced transparency is achieved for $\omega_{\mathrm{p}} / \sqrt{ } \gamma<\omega<\omega_{\mathrm{p}}$.

The purpose of this study is to assess the possibility of transmitting electromagnetic energy flux through an overdense plasma by using this induced transparency phenomenon. Numerical particle-in-cell simulations are used together with analytic stability computations.

\section{NUMERICAL SIMULATIONS OF A 1D PLASMA SLAB}

The interaction of an ultra-high intensity electromagnetic wave with a plasma slab has been initially studied in the 1D case of a normally incident plane wave. The electromagnetic field as well as the plasma remain invariant by translation in directions orthogonal to the direction of propagation. The particle-in-cell simulation takes into account the three components of particle velocities but the only spatial dimension in the direction of propagation. The Maxwell equations deal with a three components electric field and a magnetic field with two transverse components.

The plasma slab width is 10 times the vacuum wavelength $\lambda_{0}=2 \pi \mathrm{c} / \omega$. Initially the electron density is uniform with $n=1.5 n_{c^{*}}$. The particle temperature is $1 \mathrm{keV}$. A small amplitude wave is totally reflected by such a layer.
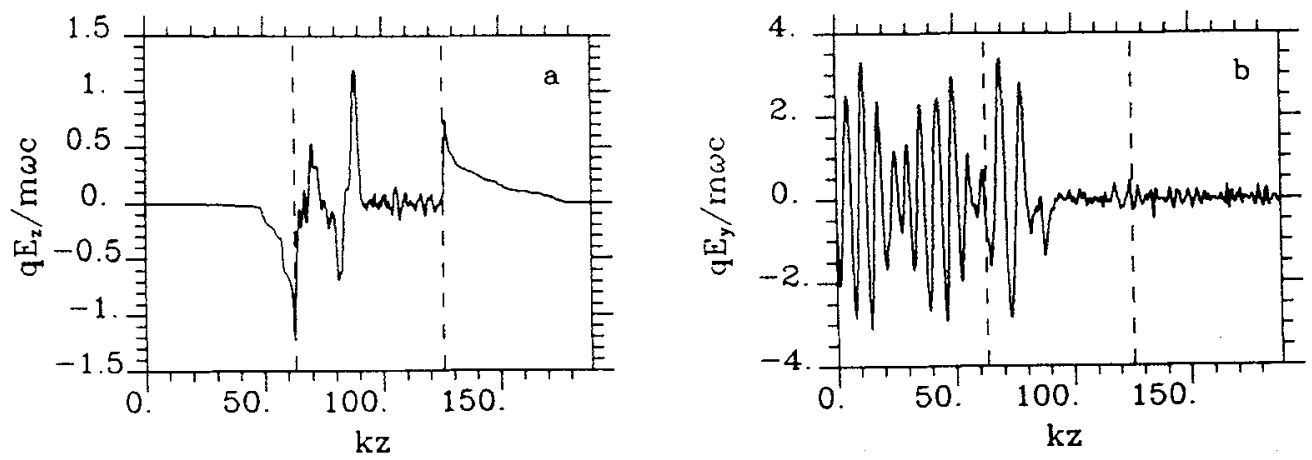

Figure 1: Electric field at time $250 \omega^{-1}$; the vertical dashed lines indicate the edges of the layer. (a) longitudinal electric field; (b) transverse electric field.

The launched wave reaches an intensity such that $\gamma=2$, where $\gamma$ is given by Eq.(2). Its rise time is 10 wave periods. At the left side of the simulation box, the polarization of the incoming wave is circular. It is seen on Fig. 1 b that the transverse electric field does propagate through the overdense plasma with intensities in the same range as the vacuum field. However several unexpected features are also displayed by the simulation. A large irregular longitudinal electric field is generated (Fig. 1a).

The electron phase space plot ( Fig. 2a) shows a strong longitudinal heating behind the wave front. As a result the absorption coefficient of the wave reaches 0.8 and the front velocity is reduced. In a cold plasma the front velocity $V_{f}$ should be given by $V_{f}=c N /\left[1+n(\gamma-1) / 2 n_{c} \gamma(\gamma+1)\right]$, where $N$ is given by Eq. (1). This expression takes into account the energy flux which is necessary to give electrons the transverse momentum corresponding to the wave amplitude. In the numerical simulation, it yields $V_{f}=0.30 \mathrm{c}$ while 
the measured one is $0.15 \mathrm{c}$. This last value is obtained when the finite reflection rate and the longitudinal heating are added in the energy flux balance. Note that the longitudinal heating lowers the effective plasma frequency so that the layer becomes almost transparent a few periods after the layer crossing.
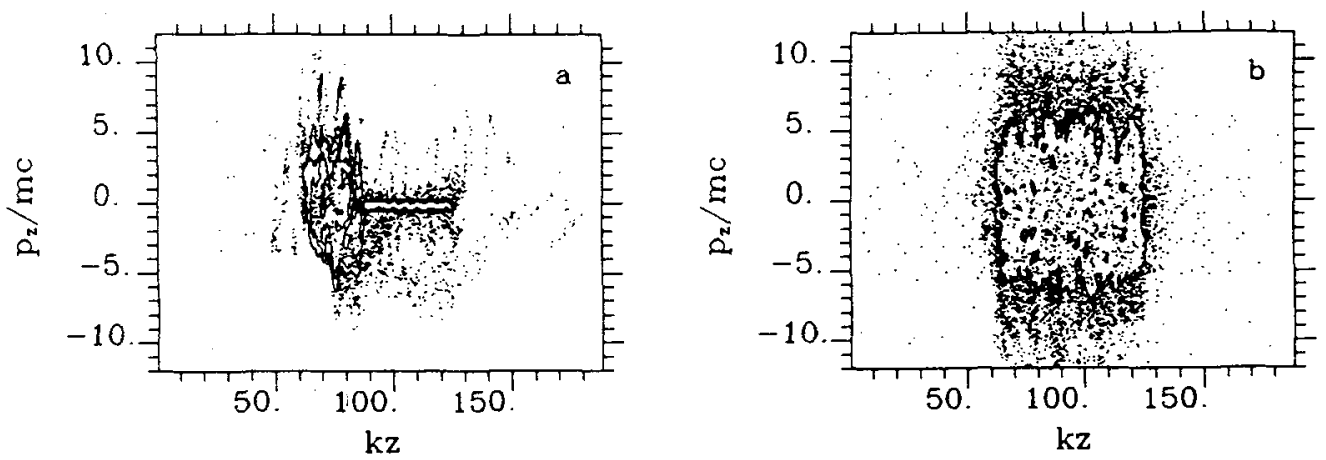

Figure 2: Electron longitudinal phase space plot. (a) $t=250 \omega^{-1}$; (b) $t=1000 \omega^{-1}$.

The strong longitudinal heating could result either from the interaction of electrons with the front of the wave as it penetrates through the cold plasma or from strong parametric instabilities excited by the intense wave as soon as it penetrates through the layer. The irregular behavior of the longitudinal electric field suggests that parametric instabilities play an important role. In order to have a more rigorous evidence of their importance, we have injected a linearly polarized wave on the left side of the simulation box. The interaction is qualitatively the same as in the case of a circularly polarized wave, but the wave does not remain polarized linearly. After a short time, it looks totally depolarized, indicating obviously that the symmetry of the incident wave has been destroyed by parametric instabilities.

After the layer crossing by the front, the wave propagates almost linearly in the plasma with a relativistic temperature (Fig. 2 b). The wave amplitude is slowly modulated and the polarization of the incident wave is restored.

\section{WAVE STABILITY IN A COLD PLASMA}

Although the parametric instabilities are more obviously identified in the numerical simulations for linear polarization, the wave stability will be studied for a circularly polarized wave. As a matter of fact, for such intensities, the usual weak coupling approximations do not apply and there is no simplifying approximations available in the general case. Fortunately, the nonlinear periodic circularly polarized wave yields an exact dispersion equation [4,5] for pertubations propagating like the periodic wave, namely for the perturbations which are taken into account in the 1D simulations. Such perturbations are characterized by the excitation of a longitudinal electric potential $\phi_{\sim} \exp [\mathrm{i}(\mathrm{Kz}-\Omega \mathrm{t})]$, and transverse components E $\exp \{\mathrm{i}[(\mathrm{K}+\mathrm{k}) \mathrm{z}-(\Omega \pm \omega) \mathrm{t}]\}$. The dispersion relation is then given by

where

$$
D_{+} D_{-} D_{p}+\frac{\omega_{p}^{2}}{\gamma^{3}}\left(\frac{e E}{m \omega c}\right)^{2}\left(D_{p}-K^{2} c^{2}\right)\left(D_{+}+D_{-}\right)=0 \text {, }
$$

$$
\begin{gathered}
\mathrm{D}_{ \pm}=(\Omega \pm \omega)^{2}-(\mathrm{K} \pm \mathrm{k})^{2} \mathrm{c}^{2}-\frac{\omega_{\mathrm{p}}^{2}}{\gamma}, \\
\mathrm{D}_{\mathrm{p}}=\Omega^{2}-\frac{\omega_{\mathrm{p}}^{2}}{\gamma} .
\end{gathered}
$$



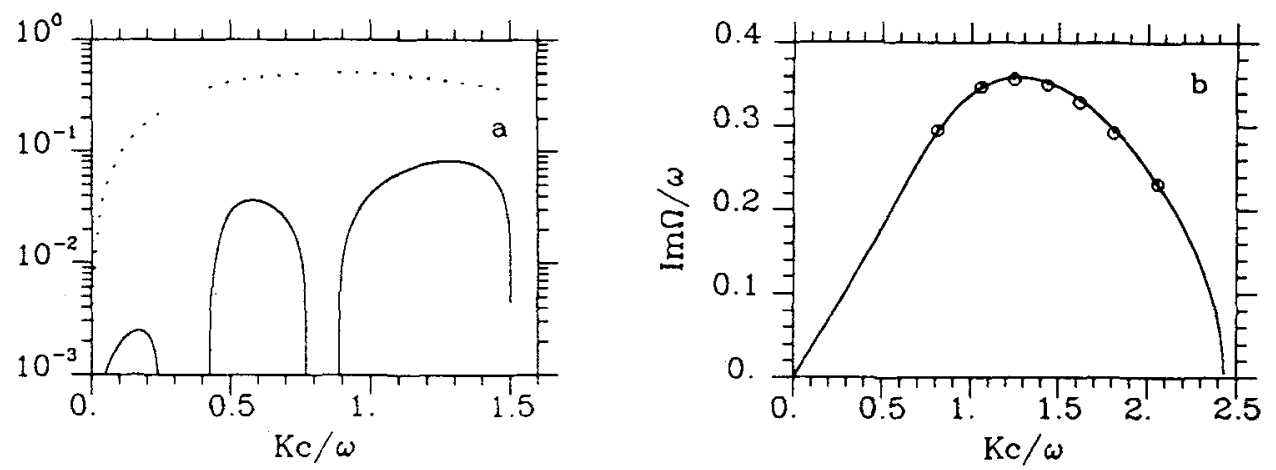

Figure 3: Growth rates $(-)$ and frequencies $(\cdots)$ of parametric instabilities given by Eq. (3). (a) $\omega_{p} / \omega=0.44$, for $\gamma=1.022$; (b) $\omega_{\mathrm{p}} / \omega=1.22$ for $\gamma=2$.

On one hand, for $\gamma=1.022$, in an underdense plasma, three parametric instabilities are found [6] (forward and backward Raman scattering, modulational instabilities). They correspond to three distinct region in K-space as shown in Fig.3a. On the other hand, Fig.3b shows the merging of these usual parametric insabilities in the overdense plasma to yield a continuous unstable domain extending from $\mathrm{K}=0$ to a maximum $\mathrm{K}$ larger than $\mathrm{k}$. In this last case, the maximum growth rate reaches $0.4 \omega$. The instability is found to be absolute in the wave frame with a similar growth rates and a wave number close to $\mathrm{k}$. Consequently these perturbations are generated at the wave front over a short distance. It is a possible explanation of the observed heating.

\section{WAVE STABILITY IN A HOT PLASMA}

It has to be understood why the instabilities do not appear after the crossing of the layer by the wave front. In order to look for such an explanation, the stability has been studied when the wave propagates in plasma with a longitudinal electron momentum spread.

In such a plasma, a large amplitude circularly polarized electromagnetic wave propagates also sinusoidally without inducing any longitudinal electric field. Its propagation may be characterized by a refraction index $\mathrm{N}$ which is now

$$
\mathrm{N}^{2}=\frac{\mathrm{k}^{2} \mathrm{c}^{2}}{\omega^{2}}=1-\frac{\omega_{\mathrm{p}}^{2}}{\omega^{2}}<\frac{1}{\gamma}>
$$

where $\gamma$ is the electron Lorentz factor and the averaging is taken over the electron momentum distribution function.

The dispersion equation for the perturbation can also be obtained in this case [5]. Its discussion shows a reduction of the growth rates as the longitudinal momentum spread $\Delta p$ increases. This stabilization is more easily seen by using a $1 \mathrm{D}$ particle-in-cell numerical simulation. At $t=0$, a large amplitude periodic circularly polarized electromagnetic wave is launched. For a cold plasma, the longitudinal field amplitude grows according to the solutions of Eq.(3) as shown on Fig.3b where the circles correspond to the measured growth rates in the simulation.

The growth rates are significantly reduced provided that the longitudinal momentum spreading exceeds mc (Fig.4). This result explains the attenuation of the parametric instabilities after the layer crossing, since the observed longitudinal momentum spreading is then larger than mc. 


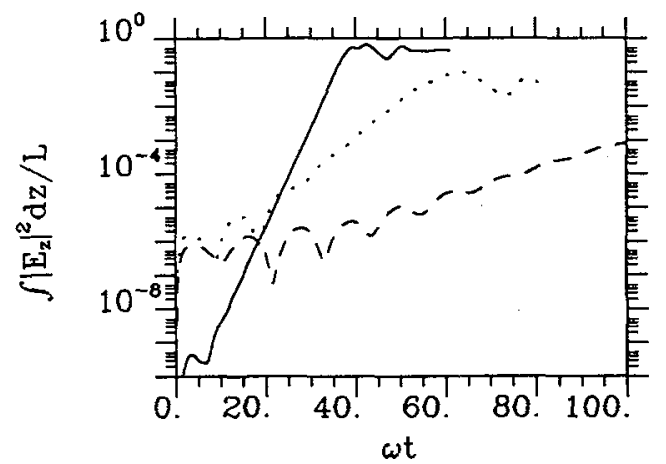

Figure 4: Longitudinal electric energy density versus time for $\omega_{\mathrm{p}} / \omega=1.22, \gamma=2, \Delta \mathrm{p} / \mathrm{mc}=0.005$ (-), $\Delta \mathrm{p} / \mathrm{mc}=1.152 \quad(\cdots), \Delta \mathrm{p} / \mathrm{mc}=3.4(--)$.

\section{NUMERICAL SIMULATION OF A 2D PLASMA SLAB}

In order to describe transverse filamentation and self-focusing of the incident beam, a particle-in-cell numerical simulation with the same parameters as in Sec.(2) has been performed with a $2 \mathrm{D}$ code. The incident wave was linearly polarized and the code did not allow depolarization effects. After averaging over the transverse direction, the wave behavior and the plasma evolution did not differ markedly from the 1D case. The main new feature appears on Fig. 5, showing a wave filamentation as well as a strong transverse modulation of the plasma density. Additionally the electron heating occurs also in the transverse direction. Note however that the transmission rate decreases quickly as the width of the plasma layer increases, due to a strong plasma absorption, an effect already present in $1 \mathrm{D}$ simulations but to a lower extent.
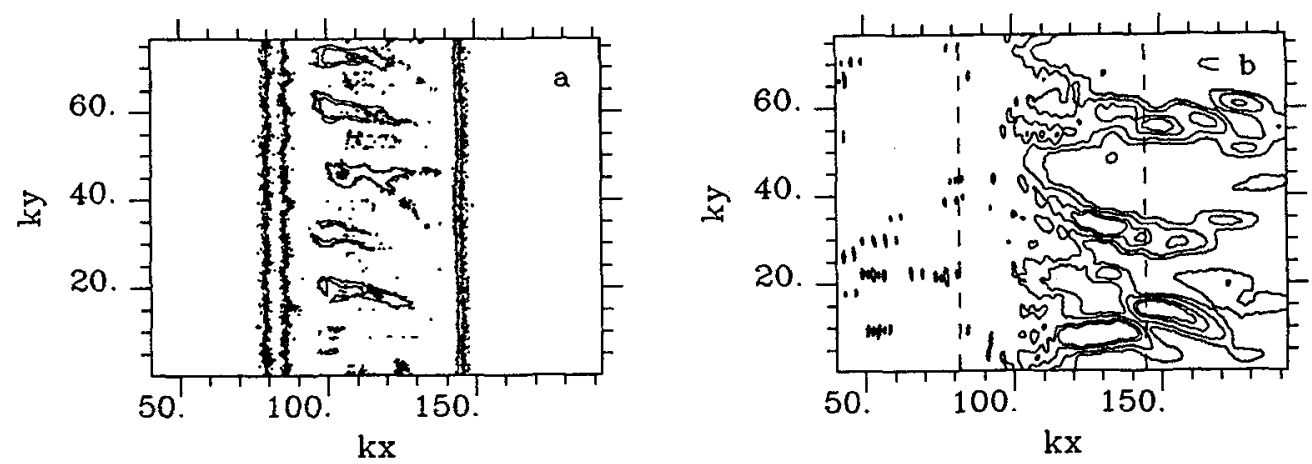

Figure 5: (a) Plasma density and (b) transverse electric energy density at time $t=400 \omega^{-1}$ in the $2 D$ simulation for a plane incident wave.

In order to simulate the interaction of a focused laser radiation with the layer, a finite width gaussian beam has been launched on the left side of the simulation box (Fig. 6). The peak intensity is the same as the intensity in the previous cases. The beam is about 10 wavelength wide. A strong self-focusing is apparent on Fig. $6 \mathrm{~b}$ since the beam emerges at the right edge of the layer with a three times smaller width and a three times higher intensity. In cylindrical geometry, this enhancement of the intensity would be even stronger. The light propagates in a hot channel in which the density is lower than the initial one (Fig. 6a). The plasma has been partially ejected laterally and two dense ridges appear along the edges of the channel. 

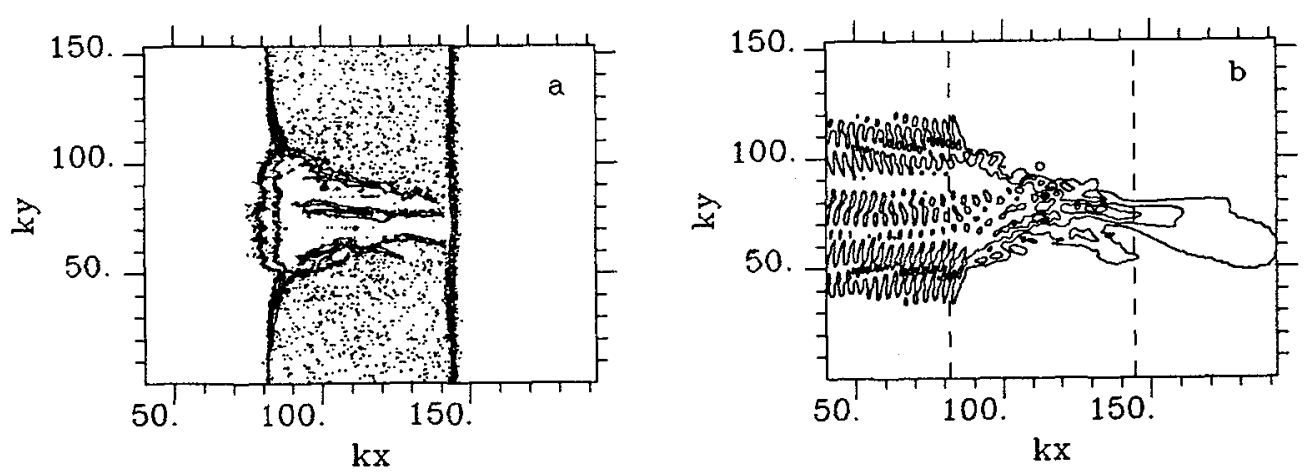

Figure 6: (a) Plasma density and (b) transverse electric energy density at time $t=400 \omega^{-1}$ in the $2 \mathrm{D}$ simulation for a focused incident beam.

The plasma density profile looks somewhat similar to the hole bored in a very overdense plasma by a focused beam [7]. However the mechanisms are quite different. In the very overdense case, the light is not intense enough to induce transparency. In order to drill its hole, the light has to push the plasma electrons and ions so that the front velocity remains small compared with the velocity of light. If the intensity induces transparency of the overdense plasma, the wave front propagates much faster as the wave has to heat the electrons but is not slowered by the ion inertia.

\section{CONCLUSIONS}

A relativistic electromagnetic wave propagates through an overdense plasma. A large fraction of the incident energy flux is converted into electron thermal longitudinal motion. The origin of this heating could be the fast growing parametric instabilities which occur in a cold plasma and are stabilized in a relativistically hot plasma.Filamentation occurs in the transverse direction for an incident plane wave. For a beam with a limited transverse extension, self-focusing reduces its width to that of a thin intense filament within only a few wavelength. This filament has a higher intensity and propagates further into the dense plasma. Applications of these physical effects can be found in the generation of high energy particles and in processes where it is needed to inject intense radiation through an overdense plasma.

\section{REFERENCES}

[1] M. Pessot, J.Squier, G. Mourou, and D.J. Harter, Opt. Lett. 14, 797 (1989); M. Pessot, J.Squier, P. Bado, G. Mourou, and D.J. Harter, J. Quantum Electron. QE-25, 61 (1989); J.P. Watteau, G. Bonnaud, J. Coutant, P. Dautray, A. Decoster, M. Louis-Jaquet, J. Ouvry, J. Sauteret, S. Seznec and D. Teychenné, Phys. Fluids B4, 2217 (1992).

[2] A.I. Akhiezer and R.V. Polovin, Zh. Eksp. Teor. Fiz. 30, 915 (1956) [Sov. Phys. JETP 2,696 (1958)]; P. Kaw and J. Dawson, Phys. Fluids 13, 472 (1970); C. Max and F. Perkins, Phys. Rev. Lett. 27, 1342 (1971).

[3] F.S. Felber and J.H. Marburger, Phys. Rev. Lett. 36, 1176 (1976).

[4] A. S. Sakharov and V. I. Kirsanov, Phys. Rev. E 49, 3274 (1994).

[5] S. Guérin, J.C. Adam, A. Héron, G. Laval, P. Mora, A. Bendib, Phys. Plasmas 2, 2807 (1995).

[6] C.J. McKinstrie and R. Bingham, Phys. Fluids B 5, 2626 (1992).

[7] S.C. Wilks, W.L. Kruer, M. Tabak and A.B. Langdon, Phys. Rev. Lett. 69, 1383 (1992), 